\title{
Optimal Aerodynamic Shape Optimization of a Paraglider Airfoil Based on the Sharknose Concept
}

\author{
Amir A. Abdelqodus ${ }^{1, a}$ and Innokentiy A. Kursakov ${ }^{1, b}$ \\ ${ }^{1}$ Moscow Institute of Physics and Technology, 140180, Zhukovsky, Russia
}

\begin{abstract}
The effects of classical air inlet configurations on the aerodynamic performance of a paraglider airfoil are firstly presented. Followed by conducting gradient-based aerodynamic shape optimization of the baseline airfoil used in the investigation of the classical air inlets. Different air inlet configurations are introduced to the optimized profile, and there effects on the aerodynamic performance are then compared to the initial and optimized airfoils. The Reynolds averaged Navier-Stokes equations (RANS) are solved for the flow field around the closed and open airfoils. The canopy is assumed to be smooth, rigid and impermeable. Results are focused on both of lift and drag coefficients for performance analysis and on the internal pressure coefficient which can be critical for a real flexible wing regarding the risk of collapse.
\end{abstract}

\section{Introduction}

Maintaining a good internal pressure at a high angle of attack offers, among other things, longer brake travel and increased stall resistance at lower speeds. Therefore one needs to place the air intake at or close to the stagnation point in order to get higher internal pressure than the external pressure at every point on the airfoil surface. Unfortunately the stagnation point is not fixed, but it varies with the angle of attack.

One way to fix this, is to design the air inlet or simply inlet, with the novel SharkNose shape, which was developed and patented by the company "Ozone" [1]. It adds a concave part in the usual range of the stagnation point. This concave part will greatly reduce the range size of the stagnation point, and shall be considered as an area where the airflow slows down. It produces the opposite effect to a venturi by presenting a larger area where the air will flow slower, knowing that as the airflow slows down in a given area, the closer to unity the pressure coefficient, $\mathrm{C}_{\mathrm{p}}$, of this area will be. The symmetrical shape, added to a rounded profile enables satisfactory behavior at both low and high angles of attack without added drag. Moreover, with the stagnation being less mobile, it is possible to reduce the size of the intake and therefore to obtain an even more pressure in front of it.

The airfoil presented in this paper is a custom paraglider airfoil, which has a chord length of $3 \mathrm{~m}$ and flies at a cruise speed about $10 \mathrm{~m} / \mathrm{s}$. The angle of attack varies from relatively low to high values, representing accelerated and normal flights, respectively. The main objectives are to quantify the influence of different classical inlets on the aerodynamic performance, and find the optimal aerodynamic design shape of the inlet to reduce the decrease in performance due to the classical inlets.

\section{Numerical methodology}

The open-source SU2 code $[2,3]$ is used to perform the flow analysis, and gradient-based optimal shape design for the paraglider airfoils. The 2-D simulation of the cross section of the airflow around a paraglider airfoil was obtained by solving the steady-state RANS over a Ctopology flow domain. Domain boundaries for this case are placed 150 chord lengths from the airfoil surface to minimize the effect of the far-field boundaries on the solution. The flow is considered incompressible with constant properties. For the free stream Reynolds Number of $2.086 \times 10^{6}$, the turbulent Reynolds stresses were taken into account by choosing the Spalart-Allmaras (S-A) [4] turbulence model. Mesh spacing at the airfoil boundary $2 \times 10^{-6}$ to ensure $y+<1$ everywhere over the airfoil surface. Due to practical constraints, the interior of the paraglider is solved using the same turbulence model, regardless of the low Reynolds number in this area for which this model may not be well adapted.

The closed and paraglider airfoil geometries were imported into the mesh generator software Pointwise $\mathbb{}$. Over the closed airfoils, two-dimensional discretized volumes consisting of quadrilateral elements conforming to the airfoil surface are generated. For the open airfoils, the discretized volume consists of hybrid meshes conforming to the airfoil surface. Structured meshes are used to analyze the boundary layer in the proximity of the canopy and the outer domain. While unstructured meshes are created for the interior domain of the open airfoils with mostly quadrilateral cells. The interior mesh

a Corresponding Author: a.amir@phystech.edu bi.kursakov@phystech.edu 
characteristics are adapted to the node distribution of the external airfoil surface's mesh.

Roe's second-order upwind scheme is used to calculate the convective fluxes. Venkatakrishnan's limiter is applied to the primitive variables. Turbulent variables for the S-A model are convected using a second-order scalar upwind method, and the viscous fluxes are calculated using the corrected average-gradient method. Implicit, local time-stepping is used to converge the problem to the steady-state solution, and the linear system is solved using the GMRES method [5] with a maximum error tolerance of $\mathcal{O}\left(10^{-6}\right)$ for each nonlinear iteration of the flow solver. Characteristicbased far-field boundary conditions are applied to the outer domain boundaries, and an adiabatic wall boundary condition is enforced on the airfoil surface.

\section{Classical inlet configurations}

Two inlet configurations will be presented in this section. The first inlet is created using a cut in the pressure side of the airfoil. The second inlet is an offset inlet applied to the baseline airfoil with the same width as the first inlet. Both inlet shapes and sizes are used in industry.

A near view of the leading edge of the baseline airfoil with the plain cut, or inlet 1 for short, and with offset inlet, or inlet 2 are shown in Fig. 1a and 1b, respectively, with cut dimensions of $3 \% \mathrm{c}$ width each. A view of the mesh around the whole closed and open airfoils with inlet 2 are shown in Fig. $2 a$ and 2b, respectively.

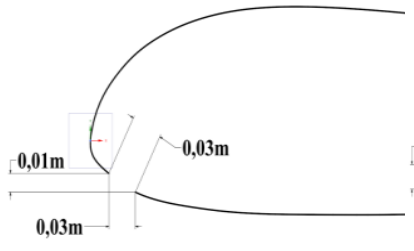

(a)

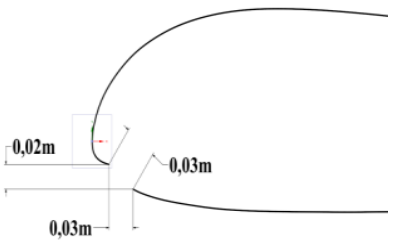

(b)
Figure 1. Baseline airfoil with inlet (a) 1 and (b) 2 configurations.

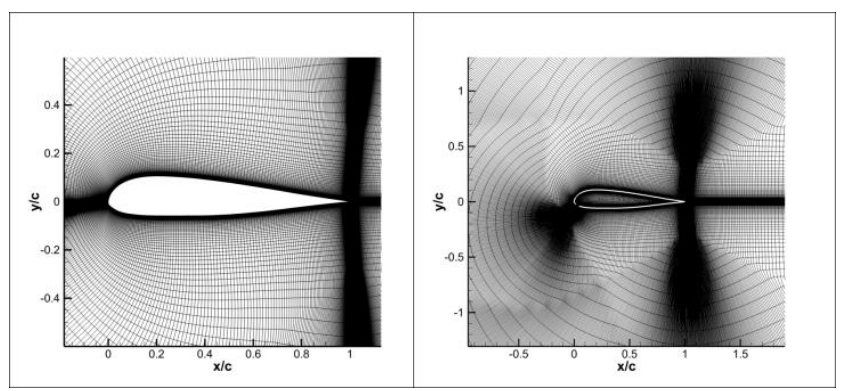

(a) (b)
Figure 2. Baseline (a) closed airfoil and (b) open with inlet 2 (airfoil in white).

The effects of the two inlet configurations on the aerodynamic performance compared to the closed airfoil are displayed in Fig. 3.

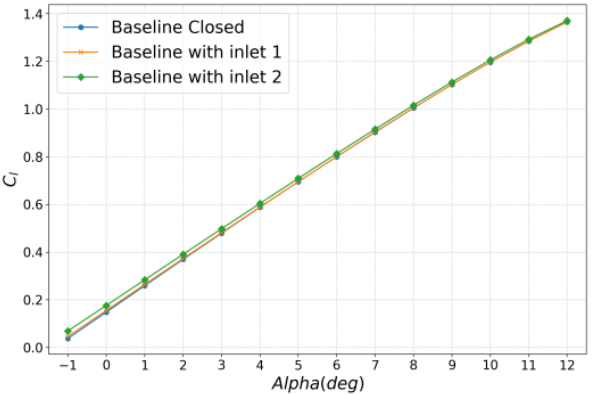

(a) Lift coefficient curves

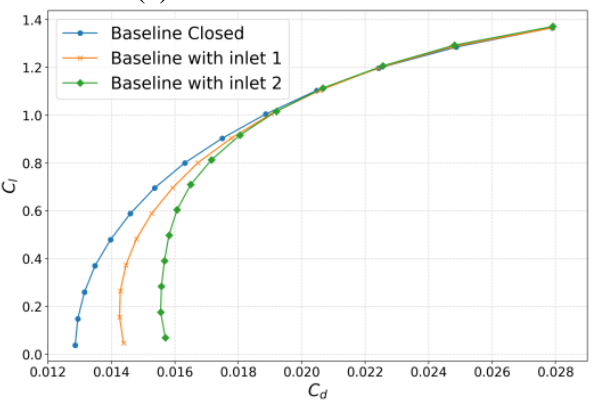

(b) Drag polar

Figure 3. The effects of the two classical inlets on the aerodynamic performance.

The increase in drag moving on from the closed, to inlet 1 then further increase with inlet 2 is due to increasing the size of the separation bubble near the opening on the lower edge for both inlets, increasing the thickness of the boundary layer than for the baseline airfoil. Additionally, a second separation bubble appears on the upper edge near the opening of inlet 2 at low angles of attack. Whereas at higher angles of attack, this effect becomes less prominent, where the curves converge with increasing angles of attack. The flow has a better orientation with the inlet, thus, the air flows directly through it, and the additional separation bubble on the upper edge of inlet 2 vanishes. This is illustrated through the streamlines for inlet 1 and 2 in Fig. 4 and 5, respectively, showing also the movement of the stagnation point position for both inlets, at angles of attack of $3^{\circ}$ and $9^{\circ}$, representing the accelerated and normal flights, respectively.

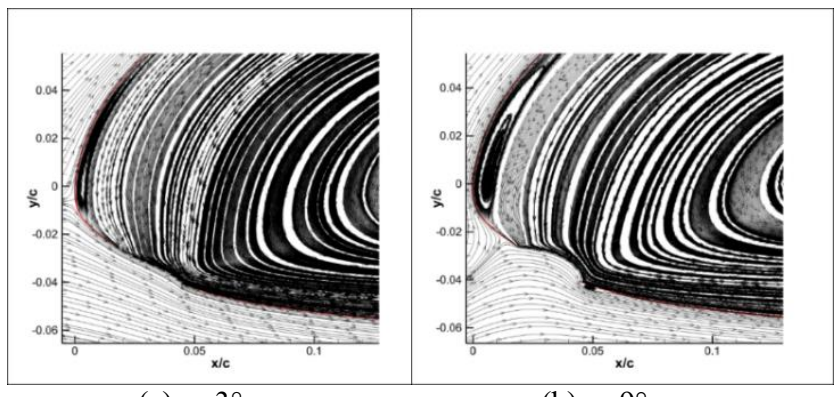

(a) $\alpha=3^{\circ}$

(b) $\alpha=9^{\circ}$

Figure 4. Streamlines inside and around airfoil with inlet 1 (airfoil edge in red). 


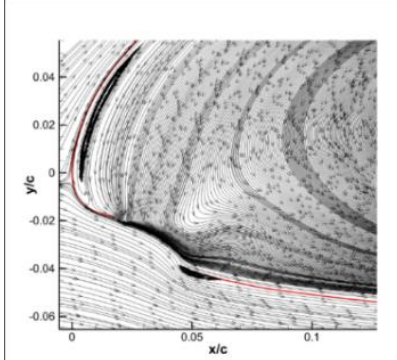

(a) $\alpha=3^{\circ}$

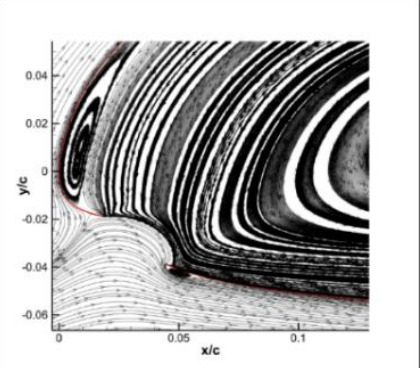

(b) $\alpha=9^{\circ}$
Figure 5. Streamlines inside and around airfoil with inlet 2 (airfoil edge in red).

The internal pressure of the baseline airfoil due to inlet 1 and 2 are shown in Fig. 6. The loss in internal pressure with inlet 1 at low angles of attack, is due to positioning the inlet downstream, increasing its distance from the stagnation point (Fig. 4a), with no offset in the lower edge. As a consequence, when the flow reaches the air inlet, it has a larger distance to accelerate and has become tangent to the canopy. Resulting in a reduction in the deflection of the fluid penetrating the canopy.

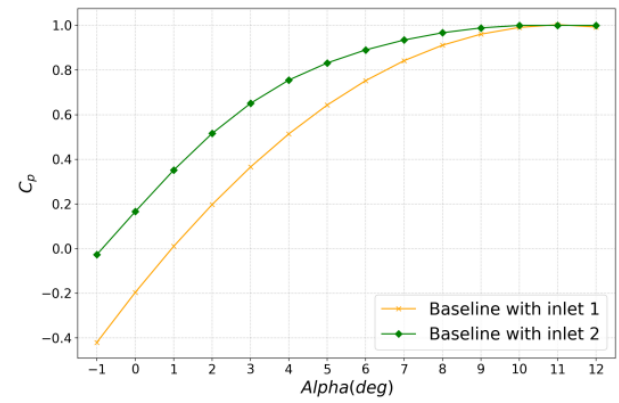

Figure 6. Internal pressure of the baseline airfoil due to classical inlets 1 and 2 .

\section{Airfoil optimization}

The methodology and results for a constrained optimal shape design of a two-dimensional airfoil at viscous turbulent flow are presented here. The optimization goal is to increase the surface static pressure on the inlet position of a modified baseline airfoil, over the atmospheric pressure at $0^{\circ}$ angle of attack, to ensure that the internal pressure of the paraglider will always satisfy the stability condition of the paraglider over all the range of flight. The maximum thickness is constrained to be greater than or equal to the initial airfoil maximum thickness which is equal to $0.164 \mathrm{c}$.

Firstly, the closed baseline airfoil geometry is modified using the open-source FreeCAD package to roughly resemble the desired SharkNose shape, to aid the optimizer in reaching the optimal required shape of the inlet area. Figure $7 \mathrm{a}$ shows the modification which is applied on the lower surface near the leading of the baseline geometry. The resulting surface pressure coefficient compared with the baseline Spirit airfoil is shown in Fig. $7 \mathrm{~b}$ at $0^{\circ}$ angle of attack, achieving a surface pressure higher than the atmospheric pressure at a low angle of attack.

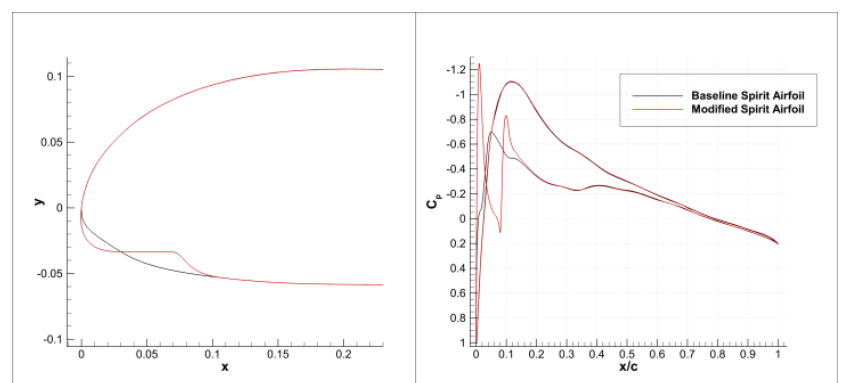

(a)

(b)

Figure 7. Modified vs Baseline airfoils.

The 2D Free-Form Deformation (FFD) [6] control point is employed as the parameterization method and the FFD box has a rectangular shape and is defined by four boundaries. which are listed in Table 1. In order to properly capture the curvature of the inlet area, a value of $0.01 \mathrm{c}$ is chosen for the uniform spacing of the control points on the surface of the FFD box. Accordingly, the FFD is created with control point dimensions of 102 by 1 in the $\mathrm{x}-$, and $\mathrm{y}$-directions, respectively. Only the lower surface of the airfoil is allowed to deform, and only the control points surrounding the inlet area on the lower surface of FFD box are set as design variables, and the rest are held fixed. Hence, the number of design variable are 9. The lower and upper bounds for each design variable is set to $0.05 \mathrm{c}$. To ensure that the deformed geometry does not contain any jumps or kinks. The optimization tolerance is set to $1 \times 10^{-6}$ for the KarushKuhn-Tucker (KKT) condition [7, 8].

Table 1. FFD box definition.

\begin{tabular}{|l|c|c|c|c|}
\hline & $\boldsymbol{B}_{\text {upper }}$ & $\boldsymbol{B}_{\text {lower }}$ & $\boldsymbol{B}_{\text {left }}$ & $\boldsymbol{B}_{\text {right }}$ \\
\hline FFD control point & 0.2 & -0.2 & -0.01 & 1.01 \\
\hline
\end{tabular}

Finally, a redesign of the airfoil is performed using the gradient information obtained from the adjoint formulation [2, 9]. Figure 8 displays the final deformed airfoil shape. Upon completion, the average surface pressure on the lower side was successfully increased from -10.19 to -9.88 , which is a $3 \%$ increase, and the maximum thickness of the final airfoil design met the constraint. The convergence histories of the objective function are plotted in Fig. 9.

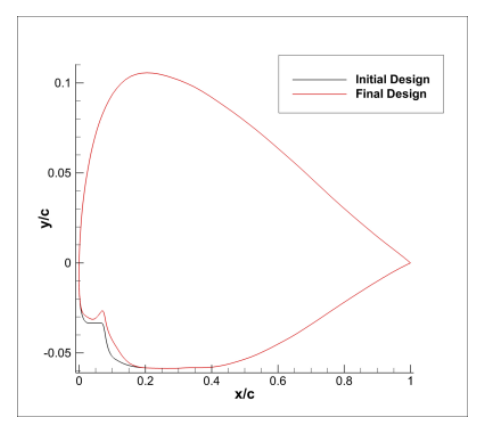

Figure 8. Deformation comparison for the initial and optimized airfoil. 


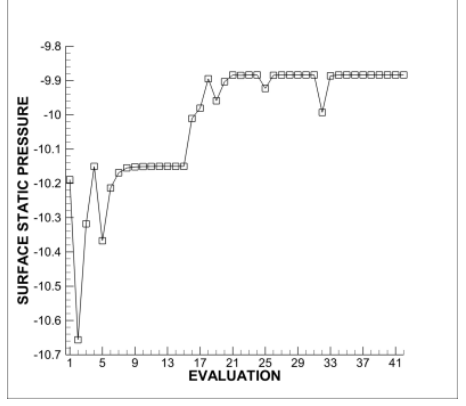

Figure 9. Convergence histories of the average surface static pressure for the modified baseline airfoil optimization.

The $\mathrm{C}_{\mathrm{p}}$ distributions of the initial and final designs (Fig. 10) show the achievement of the desired higher $C_{p}$, with smoothening the pressure distribution the at the optimized inlet area, which would also decrease the flow separation near the inlet.

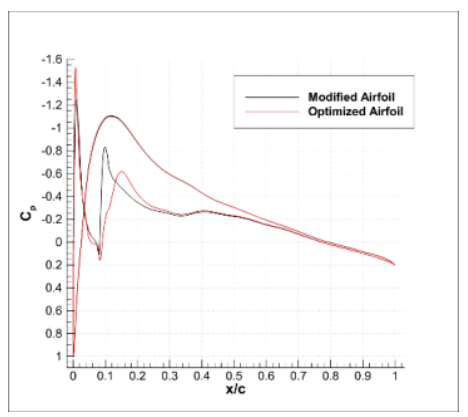

Figure 10. Surface pressure coefficient comparison for the initial and optimized airfoils at $\alpha=0^{\circ}$.

\section{Optimized air inlet configurations}

The width dimensions of the air inlets are chosen to be greater than or equal $1 \% \mathrm{c}$ to represent sport paragliders, as illustrated in Fig. 11.

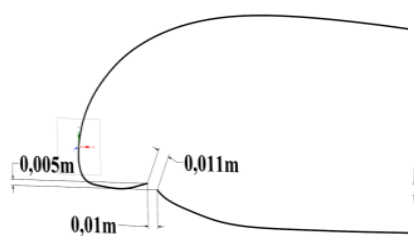

(a)

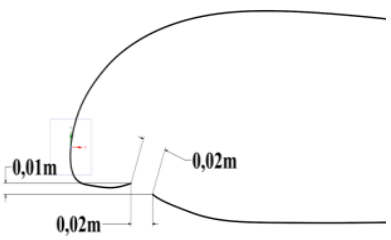

(b)
Figure 11. The optimized airfoil with inlet (a) 1 and (b) 2 configurations.

The grids used in this case have twice the tangential grid nodes conforming to the airfoil surface, which were used in the classical open airfoils case with the same number of nodes in the normal direction. Thus, the optimized airfoil is re-meshed to match the open airfoils' grids.

The effects of the two inlet configurations on the aerodynamic performance compared to the closed optimized airfoil are displayed in Fig. 12. It is shown that inlet 1 has the lesser effect on the degradation of the aerodynamic performance as expected, owing to its smaller width. For small air inlet widths, there is no difference in the slope between the optimized closed airfoil and the open airfoils. The lift curve of the paraglider airfoil with inlet 1 is almost the same as that of the baseline closed airfoil, but inlet 2 has slightly lower lift. The adverse effects on drag due to the openings are very small compared to the effects of the two classical inlets that were discussed earlier. Separation bubbles only appear on the external surface of the upper edge of the inlet. No separation occurs on the lower edge of the inlet due to its concave shape, reducing the opening effects on the rise in drag, opposing the investigated classical inlets. Inlet 2 has a slight increase in drag over inlet 1 , which is probably due to the increase of the inlet width, obscuring the flow. Even though, the separation bubbles on the external side of the upper edge of the inlets are slightly higher for the inlet with the smaller width. This is shown in Fig. 13, and Fig. 14 for inlets 1 and 2, respectively, through the streamlines at $3^{\circ}$ and $9^{\circ}$ angles of attack. Thus, the main contributor to the overall increase in drag, is the increase in inlet width, owing to the concave shape of the inlet.

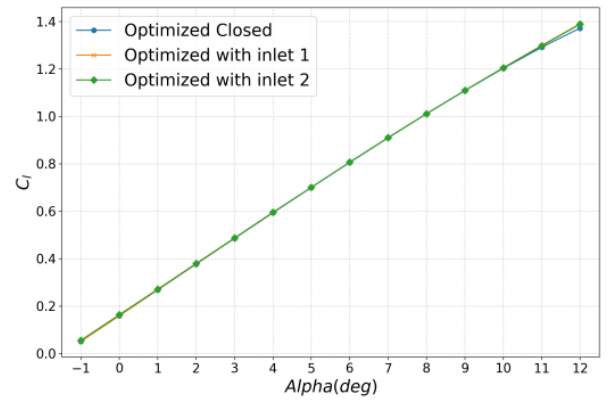

(a) Lift coefficient curves

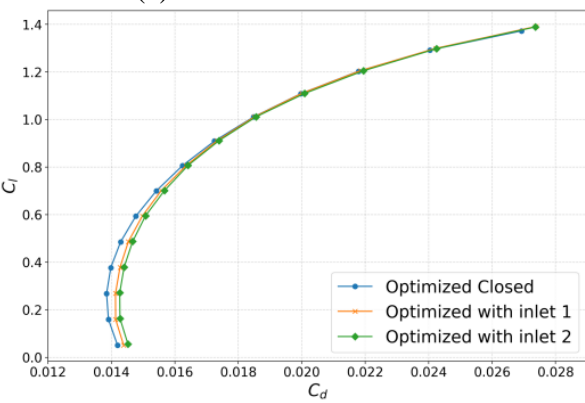

(b) Drag polar

Figure 12. The effects of the optimized inlets on the aerodynamic performance.

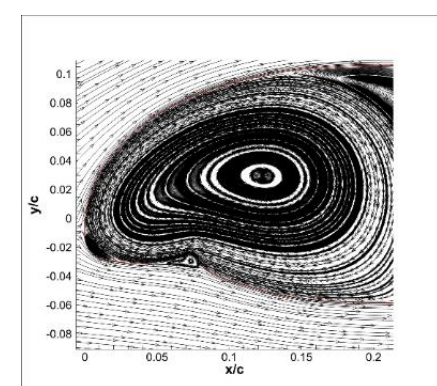

(a) $\alpha=3^{\circ}$

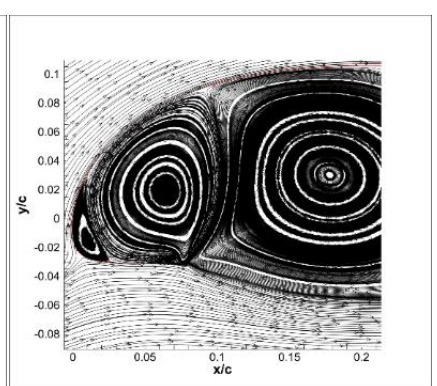

(b) $\alpha=9^{\circ}$
Figure 13. Streamlines inside and around the optimized airfoil with inlet 1 (airfoil edge in red). 


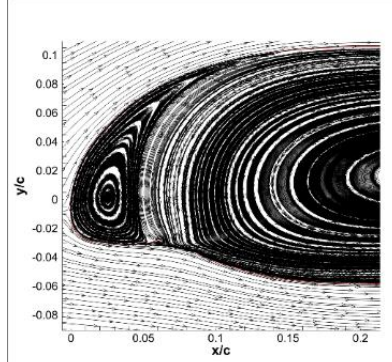

(a) $\alpha=3^{\circ}$

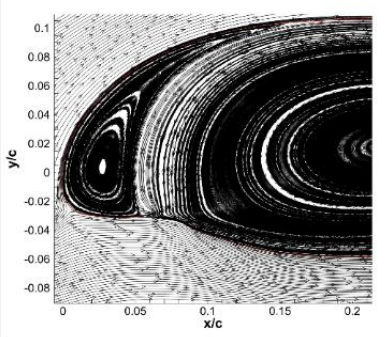

(b) $\alpha=9^{\circ}$

Figure 14. Streamlines inside and around the optimized airfoil with inlet 2 (airfoil edge in red).

The internal pressure of the optimized inlets are shown in Fig. 15. The results are close to each other, but inlet 1 achieved a slightly higher internal pressure over the flight range than inlet 2 .

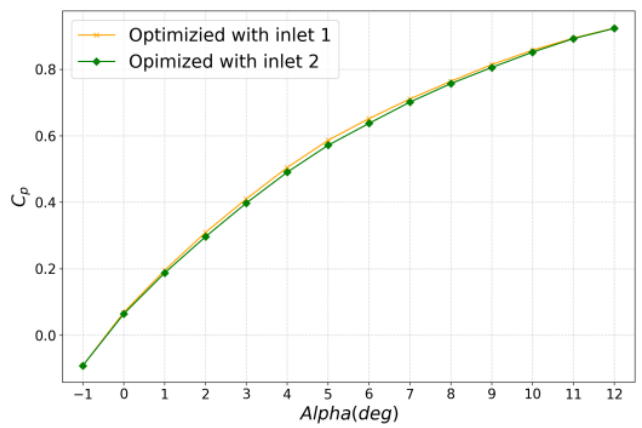

Figure 15. Internal pressure comparison of the baseline airfoil with inlet 1 and 2 .

\section{Best inlet configuration}

The classical inlet with an offset proved to have the best inflation at the whole range of flight, and has also achieved a slight increase in lift over its baseline closed profile. However, this came with the price of having the highest additional parasite drag of all the studied inlet configurations. On the other hand, both openings that were introduced to the optimized airfoil, showed balanced effects on the overall performance, with full inflation starting from $0^{\circ}$ angle of attack. Achieving a higher internal pressure than the surrounding external pressure at every point, with less adverse effects on drag and almost the same lift characteristics compared to its closed geometry. This resulted in higher glide ratio than the classical inlet by 2 counts in the normal flight regime. Additionally, the concave shape of the optimized airfoil eliminated the separation bubbles appearing on the external lower edge of both classical inlets, which not only decreased the effects of the inlet on drag by 20 drag counts compared to the classical inlet, but also it would improve recovery in case of stalling of the paraglider. As a matter of fact, the effects of an opening on the lift and drag characteristics, are less for an airfoil with the SharkNose concept, rather than introducing an inlet to a classical airfoil, as was concluded from this study, and previous studies that investigated different classical inlet configurations numerically or experimentally [10-12] to cite a few. Finally, the optimized airfoil with $1 \% \mathrm{c}$ has a slightly better overall paragliding performance than that with $2 \% \mathrm{c}$. Therefore, it is chosen as the optimal air inlet configuration for the paraglider airfoil, based on this study.

\section{References}

1. P. Frederic, A. Luc, and D. David, Ozone parapente, Institut National De La Propriété Industrielle (INPI), French patent no. 2972 422, (2011).

2. F. Palacios, M. R. Colonno, A.C. Aranake, A. Campos, S. R. Copeland, T. Economon, A. Lonkar, T. W. Lukaczyk, T. W. R. Taylor, and J. Alonso. Stanford University Unstructured (SU2): An opensource integrated computational environment for multi-physics simulation and design, (2013).

3. T. Economon, F. Palacios, S. R. Copeland, T.W. Lukaczyk, and J. Alonso. SU2: An Open-Source Suite for Multiphysics Simulation and Design. AIAA Journal, 54:1-19, (2015).

4. P. Spalart and S. Allmaras, "A one-equation turbulence model for aerodynamic flows," in 30th Aerospace Sciences Meeting and Exhibit, (1992).

5. Y. Saad and M. H. Schultz. GMRES: A Generalized Minimal Residual Algorithm for Solving Nonsymmetric Linear Systems. SIAM J. Sci. Stat. Comput., 7:856-869, (1986).

6. T. W. Sederberg and S. R. Parry. Free-form deformation of solid geometric models. In Proceedings of the 13th annual conference on Computer graphics and interactive techniques, 20:151-160, (1986). ISBN 0897911962. doi: 10.1145/15922.15903.

7. W. Karush. Minima of functions of several variables with inequalities as side conditions. Ph.d. thesis, (1939).

8. H. W. Kuhn and A.W Tucker. Nonlinear Programming. Proceedings of the Second Symposium on Mathematical Statistics and Probability, (1951). ISSN 01605682. doi: 10.1007/BF01582292.

9. T. Albring, M. Sagebaum, and N. R. Gauger. Efficient Aerodynamic Design using the Discrete Adjoint Method in SU2. AIAA 2016-3518.

10. G.M. Ware and H. J.L., Wind Tunnel Investigations of Ram-Air Inflated All- Flexible Wings of Aspect ratio 1.0 to 3.0. NASA TMSX-1923 (1969).

11. J. Ross, "Computational Aerodynamics in the Design and Analysis of Ram-Air-Inflated Wings," in RAeS/AIAA 12th Aerodynamic Decelerator Systems Technology Conference, AIAA Paper 93-1228 (Reston, VA, 1993).

12. H. Belloc, V. Chapin, F. Manara, F. Sgarbossa, and A. M. Forsting. Influence of the air inlet configuration on the performances of a paraglider open airfoil. International Journal of Aerodynamics 5:83-104 (2016). 